\title{
Category specificity in early perception: face and word N170 responses differ in both lateralization and habituation properties
}

\author{
Urs Maurer ${ }^{1,2}$, Bruno Rossion $^{3}$ and Bruce D. McCandliss ${ }^{1,4 *}$ \\ 1 Sackler Institute for Developmental Psychobiology, Weill Medical College of Cornell University, New York, NY, USA \\ 2 Department of Child and Adolescent Psychiatry, University of Zurich, Zurich, Switzerland \\ ${ }^{3}$ Unité de Neurosciences Cognitives and Laboratoire de Neurophysiologie, Université Catholique de Louvain, Louvain-la-Neuve, Belgium \\ ${ }_{4}^{4}$ Department of Psychology and Human Development, Vanderbilt University, Nashville, TN, USA
}

\section{Edited by:}

Silvia A. Bunge, University of California,

Berkeley, USA

\section{Reviewed by:}

Charles A. Nelson, Harvard Medical

School, Harvard University, USA

Lutz Jaencke, University of Zurich,

Switzerland

${ }^{*}$ Correspondence:

Bruce D. McCandliss, Box 512, 230

Appleton Place, Vanderbilt University,

Nashville, TN 37203, USA.

e-mail: bruce.mccandliss@vanderbilt. edu
N170 event-related potential (ERP) responses to both faces and visual words raises questions about category specific processing mechanisms during early perception and their neural basis. Topographic differences across word and face N170s suggests a form of category specific processing in early perception - the word N170 is consistently left-lateralized, while less consistent evidence supports a right-lateralization for the face N170. Additionally, the face $\mathrm{N} 170$ shows a reduction in amplitude across consecutive individual faces, a form of habituation that might differ across studies thereby helping to explain inconsistencies in lateralization. This effect remains unexplored for visual words. The current study directly contrasts N170 responses to words and faces within the same subjects, examining both category-level habituation and lateralization effects. ERP responses to a series of different faces and words were collected under two contexts: blocks that alternated faces and words vs. pure blocks of a single category designed to induce category-level habituation. Global and occipito-temporal measures of N170 amplitude demonstrated an interaction between category (words, faces) and block context (alternating categories, same category). N170 amplitude demonstrated class-level habituation for faces but not words. Furthermore, the pure block context diminished the right-lateralization of the face N170, pointing to class-level habituation as a factor that might drive inconsistencies in findings of right-lateralization across different paradigms. No analogous effect for the word N170 was found, suggesting category specificity for this form of habituation. Taken together, topographic and habituation effects suggest distinct forms of perceptual processing drive the face N170 and the visual word form N170.

Keywords: electroencephalography, event-related potentials, N170, face processing, word processing, habituation, adaptation, lateralization

\section{INTRODUCTION}

Electrophysiological studies have shown that visual processes are specialized for certain stimulus categories within the first $200 \mathrm{~ms}$ of stimulus presentation. Such fast and specialized brain processes have most consistently been indexed by an increased occipito-temporal N170 component of the event-related potential (ERP) for faces (e.g., Bentin et al., 1996; Botzel et al., 1995; Jeffreys, 1989; for a recent review see Rossion and Jacques, 2008) and visual words (e.g., Bentin et al., 1999; Maurer et al., 2005a,b) compared to control stimuli (e.g., words compared to non-orthographic strings of symbols or forms; faces compared to nonface objects). Enhancements in N170 responses have been linked to perceptual expertise via experiments demonstrating enhanced N170 responses to pictures of birds and dogs within birdand dog experts respectively (Tanaka and Curran, 2001), or from the reduction of N170 amplitude in response to faces when processing objects of expertise concurrently (e.g., cars, Rossion et al., 2007).

Similar evidence for the role of experience in N170 enhancement has come from experiments in the reading domain. The N170 response to words written in Japanese scripts was increased in Japanese readers compared to native English speakers without experience with the script (Maurer et al., 2008; see also Wong et al., 2005 for a similar effect in Chinese), and the N170 response to visual words increased relative to control stimuli, only after children had experienced reading training and mastered basic reading skills (Maurer et al., 2006).

In addition to amplitude differences, the lateralization of the N170 can differ by category. For example, the topography of N170 responses to faces is more right-lateralized, whereas N170 to words is more left-lateralized (for a direct comparison, see Rossion et al., 2003). This pattern of differences over left and right hemispheres for N170 responses to faces and words is reported consistently enough in the literature to rule out this effect being spurious, yet enough counter examples exist to suggest experimental factors are at play that influence the lateralization of N170. These factors have not been adequately controlled across studies. For example, a right-lateralized N170 in response to face stimuli was generally reported in studies that presented faces randomly among other object classes (e.g., Bentin et al., 1996; Botzel et al., 1995; Eimer, 1998; Itier and Taylor, 2004; although see Rossion et al., 2000). In some studies that presented faces in a series 
of other faces, however, the face N170 was bilateral or showed even a left-lateralization in the group mean (Deffke et al., 2007; Schweinberger et al., 2002a,b).

Such findings suggest that the context in which a face is presented, i.e., whether it occurs among other faces or among stimuli of other object classes, may systematically influence the degree to which a face is processed in the right hemisphere. On the other hand, left-lateralization of N170 responses to visual words appears rather consistently in skilled readers, and do not show any evidence of being manipulated by context. For example, left-lateralization has been found both under conditions in which words are presented among other words (Hauk and Pulvermuller, 2004; Hauk et al., 2006; Maurer et al., 2005a,b; Simon et al., 2007) as well as when appearing randomly among control stimuli (Bentin et al., 1996, 1999; Rossion et al., 2003; Simon et al., 2004). Other studies have suggested that the degree of left-lateralization may depend on linguistic rather than contextual factors (Hauk et al., 2006; Maurer et al., 2005a).

This pattern of findings across studies provides some initial support for the hypothesis that lateralization of the N170 is categoryrelated, reflecting different perceptual encoding mechanisms applied to words versus faces (Farah, 1991). Furthermore, this lends support to the notion that a category-level habituation effect may operate differently for faces than for words, specifically leading to reduced right-lateralization for face N170 when this category is repeated within pure blocks of face stimuli. Thus the degree to which stimulus categories are repeated in rapid succession may help explain some differences reported in the topography of the face N170 and at the same time may provide additional evidence for category specific processes of perceptual encoding that underlie N170 responses.

To test these hypotheses directly, we examined whether the sequential context in which face and word stimuli are presented affects the topography of N170 component. We presented streams of unique face and word stimuli either within blocks that consisted of only one stimulus category or within blocks that alternated between these two stimulus categories. We expected that within the "blocked" condition the N170 response to faces would demonstrate category-level habituation effects - especially over the right hemisphere, relative to N170 responses to faces presented in the "alternating" condition. Furthermore, we anticipated that contrasting such context manipulations across the categories of faces versus words should reveal important differences for habituation effects across these two categories given that reading explicitly involves viewing visual words in rapid succession yet naturalistic viewing faces rarely involves rapid succession of face stimuli.

\section{MATERIALS AND METHODS PARTICIPANTS}

Eighteen volunteers ( 7 males, 11 females) who were between 19 and 30 years of age ( 23 years on average) participated in the study. They were all right-handed and spoke English as their first language, as determined by self-report. Participants provided informed consent according to a protocol approved by the Institutional Review Board of the Weill Medical College of Cornell University.

\section{PROCEDURE}

Visual word and face stimuli were either presented in blocks with just faces or just words (blocked condition), or in blocks in which face and word stimuli alternated in order (alternating condition; see Figure 1). A stimulus was shown for $250 \mathrm{~ms}$ followed by an average interstimulus interval of $1500 \mathrm{~ms}$ (jittered between 1250 and $1750 \mathrm{~ms}$ ). The stimuli consisted of 36 faces and 36 words that were presented twice in each condition. Half of the words and half of the faces appeared first in the blocked condition, the other half first in the alternating condition. Block position and sequence were counterbalanced across subjects. Although the majority of stimuli required no response, subjects were asked to press a single button in response to an occasional (11.1\%) face or word that appeared upside-down. Accuracy and reaction time data were collected for responses to these rare target stimuli.

Faces were presented at a visual angle of $1.65^{\circ} \times 2.25^{\circ}$, words at $1.45^{\circ} \times 0.55^{\circ}$. The face stimuli were color photographs adopted from a previous study (see Jacques and Rossion, 2004 for details). The word stimuli were frequent [frequency per million based on HAL corpus (Balota et al., 2007; Lund and Burgess, 1996), computed as base-10 logarithm: mean $2.2 \pm$ SD 0.6], concrete nouns, four letters in length presented in lower case.

\section{EEG RECORDING AND ANALYSIS}

We recorded 128-channel EEG data (Hydrocel net from Electrical Geodesics) initially with a Cz reference. Data were sampled at $500 \mathrm{~Hz}$ with $0.1-200 \mathrm{~Hz}$ filter settings with impedances below $50 \mathrm{k} \Omega$. Using BESA software, data from channels with excessive artifacts were excluded and replaced applying spherical spline interpolation, and eye blinks were corrected applying multiple source eye correction to minimizing topographic distortions (Berg and Scherg, 1994). The data then were digitally bandpass filtered $(0.3-30 \mathrm{~Hz})$, segmented $(-150$ to $750 \mathrm{~ms})$, artifact rejected $( \pm 80 \mathrm{uV})$ and averaged for non-target stimuli in each condition separately. Using Brain Vision Analyzer software, the averaged data were re-referenced to average reference (Lehmann and Skrandies, 1980), and baselinecorrected (100 ms prior to stimulus presentation). After computing global field power (GFP; Lehmann and Skrandies, 1980) grand mean values were computed for all conditions.

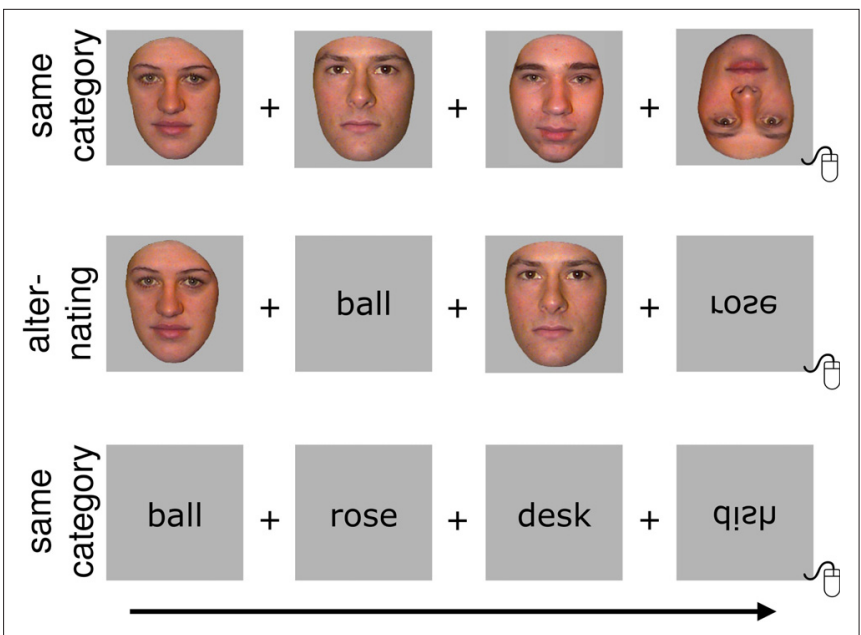

FIGURE 1 | Experimental design. Face and word stimuli were either presented in pure category blocks or in blocks alternating with stimuli from the other category. The task required subjects to press a button whenever a stimulus was presented upside-down (11.1\%). 
The resulting ERP data were analyzed in a two-step strategy. First, time windows that demonstrated habituation effects (i.e., significant differences between one-category versus two-category contexts for either faces or words) were identified for further analysis.

This first step involved computing topographic analyses of variance (TANOVA, part of the LORETA-KEY software package) on non-normalized (raw) maps contrasting the one-category versus two-category context conditions at each time point. Separate analyses were carried out for faces and words to identify time windows that would be used to explore habituation effects that might impact either category. These time point-wise TANOVAs were conducted on raw maps to detect any systematic amplitude differences between the two conditions (i.e., blocked and alternating contexts) by running a nonparametric randomization test (Holmes et al., 1996) on the GFP of difference maps between two conditions (Lehmann and Skrandies, 1980; Strik et al., 1998). An alpha level of $p<0.01$ in at least three consecutive time frames was adopted to control for multiple comparisons across these two analyses. The joint probability of $p<0.01$ in three time frames $(0.01 \times 0.01 \times 0.01)$ surpasses the Bonferroni corrected threshold of $p<0.05$ across the 450 time frames tested in these analyses $(0.05 / 450)$.

Time windows related to early perceptual processes (i.e., $<300 \mathrm{~ms}$ ) that demonstrated any effect of the context manipulation (regardless of stimulus category) were extracted in a form that was applied equally across all four conditions (faces in onecategory blocks, faces in two-category blocks, words in one-category blocks, words in two-category blocks) and subjected to two further analyses.

Since these TANOVA effects reflect habituation effects within categories, but do not inform whether the habituation effects differ between categories, additional analyses were carried out to test interactions between Context and Category. For the resulting time window we ran a MANOVA on GFP with the factors Context (blocked vs. alternating presentation) and Category (faces vs. words). To be consistent with the previously reported literature on laterality effects for N170 responses (Bentin et al., 1996, 1999; Maurer et al., 2005a,b, 2008; Rossion et al., 2003), an additional analysis focused on direct comparisons between left and right occipito-temporal channels at the negative topographic peak. The topographic peak channels were defined based on the average across all four condition grand mean values in the corresponding time segment.

\section{BEHAVIORAL ANALYSIS}

Accuracy and reaction time of responses to targets were analyzed within MANOVAs with the factors Context (one-category vs. alternating two-category blocks) and Category (faces vs. words). Mean RT was computed after excluding trials with inaccurate responses, anticipations (i.e., RT $<200 \mathrm{~ms}$ ), or unusually long delay (i.e., >3 SD slower than the mean). The range of individual subject performance (accuracy range 86-100\%; RT 469-617 ms) indicated that all subjects understood and complied with instructions, and thus no subjects were excluded from the analyses.

\section{RESULTS \\ BEHAVIOR}

Overall behavioral results of the responses to the inverted stimuli which served as rare targets demonstrated that subjects were highly accurate and attentive to the primary task (>95\% correct in all conditions). While there were no significant main effects or interactions for reaction time (i.e., all $F$-values $<1$ ), accuracy of responses revealed a subtle yet significant effect of context that was different for words and faces [Context $\times$ Category interaction, $F(1,17)=4.6, p<0.05]$. Subjects were more accurate in detecting inverted words in the context of pure blocks (words $98.3 \%$, faces $97.9 \%$ ), yet more accurate for detecting inverted faces that appeared in the mixed blocks (words 95.2\%, faces 99.3\%). No other effects were significant.

\section{TIME POINT-WISE TANOVA}

Consecutive comparisons between ERP maps elicited by faces in the blocked versus alternating conditions revealed processing differences $(p<0.01)$ within the N170 time range $(168-188 \mathrm{~ms})$, as indicated in Figure 2. The corresponding comparison between words presented in blocked versus alternating conditions revealed no processing differences within the N170 time range. Processing differences for words were only found after $300 \mathrm{~ms}$, at a sub-threshold significance level.

The time segment in the N170 in which habituation effects were found for faces was further analyzed regarding global map strength and lateralization to test whether habituation effects were specific for faces, as would be indicated by an interaction between Category (faces, words) and Context (alternating, blocked) factors.

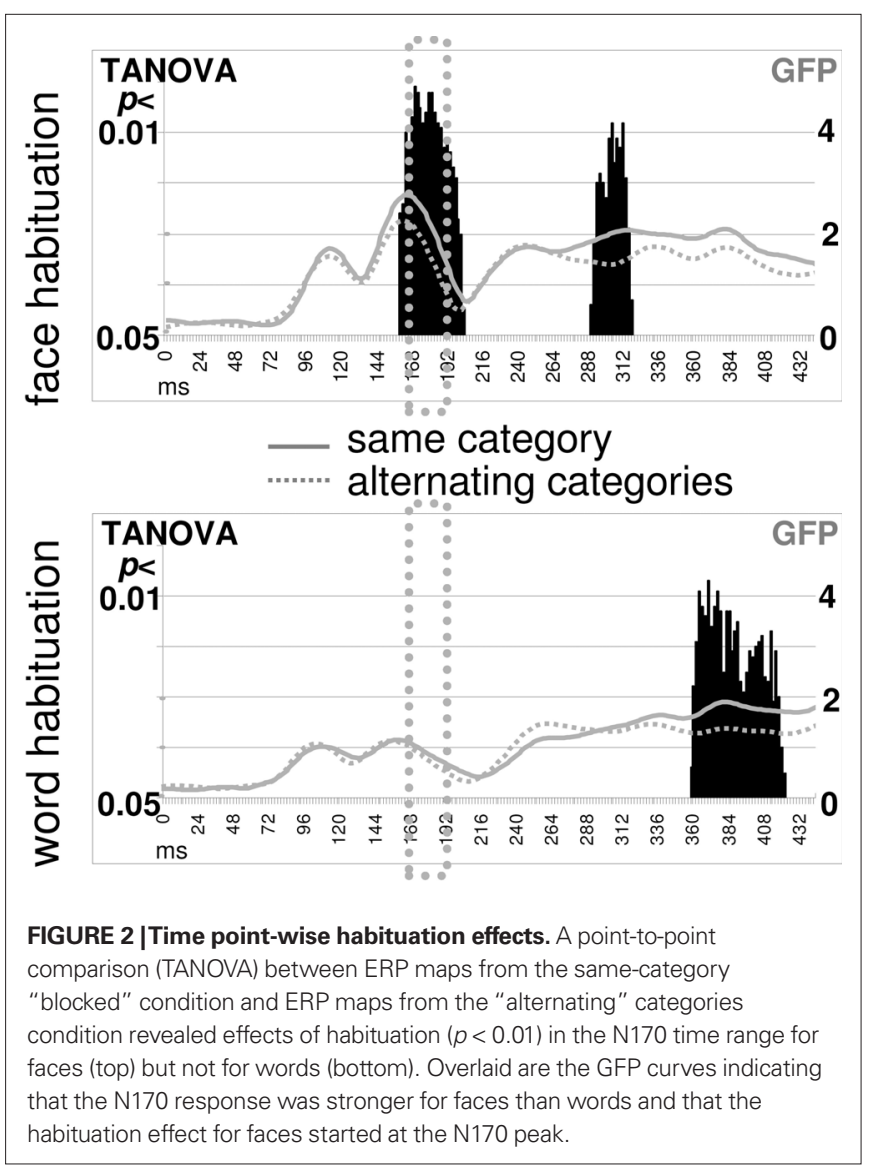




\section{GLOBAL FIELD POWER}

In the GFP analysis demonstrated significant effects for the Category $\times$ Context interaction $[F(1,17)=12.1, p<0.01]$, and also for the two associated main effects of Category $[F(1,17)=17.7, p<0.001]$ and Context $[F(1,17)=7.0, p<0.05]$. Post hoc $t$-tests revealed that faces elicited a larger N170 than words within each presentation context examined separately (blocked: $p<0.05$, alternating: $p<0.001$ ). Furthermore, post hoct-tests revealed a significant habituation effect for the amplitude of the N170 for pure blocks of faces relative to faces presented in alternating blocks $(p<0.01)$, but no such effect was present for words ( $p=0.58$; see also Figure $3 \mathrm{~A}$ ).

To test the possibility that the differential habituation effect between the two stimulus categories was driven by the overall larger
N170 response for faces compared to words, we computed the same analyses after GFP normalization for Category (division by GFP mean of blocked and alternating conditions, separately for each Category). Accordingly, the Category main effect was eliminated, and both the Context main effect $[F(1,17)=5.1, p<0.05]$ and the Context $\times$ Category interaction remained significant $[F(1,17)=8.7$, $p<0.01]$, indicating that differences in habituation between the categories were not due to overall GFP differences between face and word conditions.

\section{OCCIPITO-TEMPORAL CHANNELS}

The occipito-temporal channel analysis at the topographic peak (see also Figures 3A,B) corroborated the GFP analysis by revealing

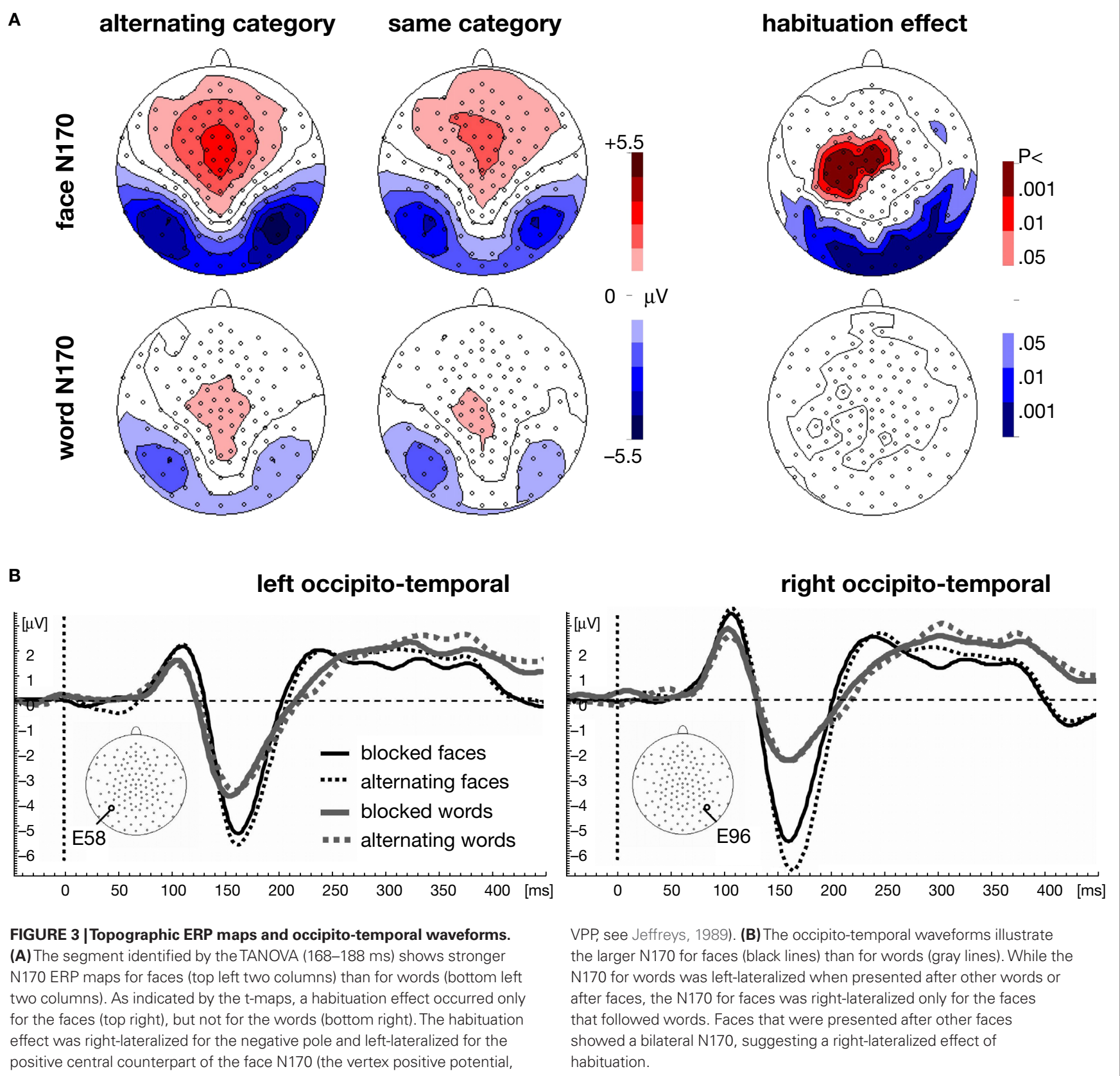


main effects for Category $[F(1,17)=48.0, p<0.001]$ and Context $[F(1,17)=14.6, p<0.01]$ as well as a Category $\times$ Context interaction $[F(1,17)=5.5, p<0.05]$. An additional Category $\times$ Hemisphere interaction indicated a more left-lateralized N170 for words compared to a more right-lateralized $\mathrm{N} 170$ for faces $[F(1,17)=5.0$, $p<0.05]$. Although the three-way interaction between Category, Context and Hemisphere resulted in only a non-significant trend $[F(1,17)=2.6, p=0.12]$, a separate analysis conducted only on face stimuli showed that the face N170 was right-lateralized with alternating presentation, but bilateral with blocked presentation [Context $\times$ Hemisphere, $F(1,17)=5.7, p<0.05]$. Finally, to test whether the lateralization difference of the habituation effect for faces would be purely topographic we did the same analysis for the faces after normalizing for GFP. Indeed, the Context $\times$ Hemisphere interaction remained significant $[F(1,17)=4.7, p<0.05]$.

\section{DISCUSSION}

The present study replicates previous findings reviewed above that visual word N170 exhibits left-lateralization and the face N170 right-lateralization, indicating different hemispheric involvement of the neural networks involved in processing these two classes of stimuli under equivalent task conditions. Most importantly, the present study demonstrated that in addition to these topographic main effects of stimulus category, the N170 response to faces and words responds differently to the sequential context in which the stimuli are presented.

The amplitude of the face N170 was strongest when each face was immediately preceded by a visual word, and the amplitude was significantly reduced (i.e., habituated) for faces preceded by other faces. This evidence of class-level habituation of the face N170 effect is in line with studies showing a reduction of the face N170 as a result of adaptation (Harris and Nakayama, 2007; Kovacs et al., 2006) and sensory competition (Jacques and Rossion, 2004). It suggests that presenting stimuli from the face category in a repetitive manner leads to similar neurophysiological effects on face processing as when a face is presented after viewing another face for an extended time period (adaptation) or when a face is presented in close spatial proximity of another face (spatial competition). The fact that such habituation effects occur across unique faces provides additional evidence that neural processes within the first $200 \mathrm{~ms}$ after presentation are sensitive to the general category of faces. Such habituation effects, however, may not only occur at the category-level, but may additionally be sensitive to information at the individual face level. This is suggested by a small N170 reduction to a face preceded by the same individual face compared to a face preceded by a different face (e.g., Itier and Taylor, 2002; Jemel et al., 2003), or by more substantial effects observed when using a long face adapter duration and a short ISI between the adapter and target faces (Jacques et al., 2007).

In addition to amplitude modulation, context effects also impacted the lateralization of the face N170. The habituation effect of the face N170 was stronger over the right hemisphere, such that faces presented among other faces were significantly less right-lateralized than faces interleaved with visual words. This novel result may help explain some of the variability across earlier studies regarding whether the face N170 was right-lateralized (Bentin et al., 1996; Itier and Taylor, 2004) or not (Deffke et al.,
2007; Schweinberger et al., 2002a,b). This suggests that the same aspect of face processing that leads to the typical right-lateralized N170 topography is also prone to habituation. Habituation paradigms thus may serve as a tool to further understand the nature of right-lateralization in face processing.

A second aim of the study was to investigate whether such habituation effects are a general property of all N170 eliciting stimuli, or whether the processes of habituation that influences the face N170 may play out differently across other categories of stimuli known to induce N170 responses. In stark contrast to the face N170 results, the visual word N170 was unaffected by alternating versus blocked contexts. This null effect, in the context of the current study, drives a significant interaction between category and context, thereby demonstrating that N170 processes associated for faces and words are differentiated not just by patterns of lateralization, but also by patterns of habituation. This suggests habituation of the N170 reflects a functional difference between fast, specialized processing of words and faces rather than some more general process that applies equivalently across these classes of stimuli.

Future research will be required to further probe the nature of these functional differences, which amount to a form of category specific processing. Although the current study was not designed to differentiate the nature of such differences, there are multiple dimensions that distinguish early processing of words and faces in the current experiment which might be investigated. First, it is possible that top-down, block level factors were not fully equated across the three central conditions of this experiment. For example, detecting inverted words within entire blocks of words may require a different degree of attention than detecting inverted faces within entire blocks of faces. The accuracy and reaction time data in this study, however, does not support a task difficulty effect between entire blocks of words and faces, although it is possible that more sensitive measures might uncover such differences.

A second more general factor that could differentiate words and faces might involve differences in processing novel items versus frequently encountered items. Note that in the current experiment familiar categories of facial features formed novel faces, yet familiar categories of letters formed words of relatively high frequency and familiarity. However, it is unlikely that the novelty/familiarity of word forms is what accounts for the observed N170 habituation differences between faces and words. Indeed, the current finding of block context impacting faces but not words has recently been replicated in a study that extended our design to include contrasts between faces and novel non-words (Mercure et al., personal communication) thereby ruling out word level familiarity as a critical factor.

Another avenue to explore might examine lower level visual factors that could be thought to interact with block context. Face information typically consists of relatively low spatial frequency information compared to visual words - a factor which is thought to contribute to the right-lateralization of faces (Sergent, 1985) but also potentially results in greater spatial overlap between successive presentations of face stimuli. This explanation, however, may be less likely, as a reduction of the face N170 can also be obtained with line-drawings of faces which consist of little low-frequency information (Harris and Nakayama, 2007). 
Alternatively, these effects may be driven by factors that are more intricately related to differences in the way these two classes of stimuli are learned. While reading typically involves rapid sequential processing of many stimuli, each of which fall within the category of visual words, habituation within blocks of visual word stimulation would be at odds with the functional goals of fluent reading. Naturalistic face processing, on the other hand, may rarely require processing of multiple faces presented in rapid succession. Thus, within this category of stimuli, category-level habituation may not interfere with overall processing goals. At the same time, it is possible that category-level habituation of structural features across faces may serve to enhance function within this domain. If naturalistic face processing typically involves looking at a face for an extended period of time, habituation to information important for classification of a stimulus as a face may facilitate the detection of more subtle changes within a face, as in the case of tracking facial expressions over time. Thus, both the presence of face N170 habituation for faces and its absence for words may help meet specific processing demands unique to processes applied to each category of stimuli.

In conclusion, it appears that in addition to lateralization differences often reported between face N170 and visual word N170 topographies, N170 responses for each of these categories reflect early perceptual processes that can be differentiated by their habituation profiles. Whatever parallels may be drawn between face and visual word N170 phenomena (for review, see McCandliss et al., 2003), clear differences between these categories must be accounted for during the early phases of perceptual encoding.

\section{ACKNOWLEDGEMENTS}

This research was supported by the Swiss National Science Foundation (Fellowship for Prospective Researchers: UM), and the US National Science Foundation (NSF 529112: BDM). We thank Nicholas Hindy for assistance in data collection.

\section{REFERENCES}

Balota, D. A., Yap, M. J., Cortese, M. J., Hutchison, K. A., Kessler, B., Loftis, B., Neely, J. H., Nelson, D. L., Simpson, G. B., and Treiman, R. (2007). The English Lexicon Project. Behav. Res. Methods 39, 445-459.

Bentin, S., Allison, T., Puce, A., and Perez, E. (1996). Electrophysiological studies of face perception in humans. J. Cogn. Neurosci. 8, 551-565.

Bentin, S., Mouchetant-Rostaing, Y., Giard, M. H., Echallier, J. F., and Pernier, J. (1999). ERP manifestations of processing printed words at different psycholinguistic levels: time course and scalp distribution. J. Cogn. Neurosci. 11, 235-260.

Berg, P., and Scherg, M. (1994). A multiple source approach to the correction of eye artifacts. Electroencephalogr. Clin. Neurophysiol. 90, 229-241.

Botzel, K., Schulze, S., and Stodieck, S.R. (1995). Scalp topography and analysis of intracranial sources of faceevoked potentials. Exp. Brain Res. 104, 135-143.

Deffke, I., Sander, T., Heidenreich, J., Sommer, W., Curio, G., Trahms, L., and Lueschow, A. (2007). MEG/EEG sources of the 170-ms response to faces are co-localized in the fusiform gyrus. Neuroimage 35, 1495-1501.

Eimer, M. (1998). Does the face-specific N170 component reflect the activity of a specialized eye processor? Neuroreport 9, 2945-2948.

Farah, M. J. (1991). Patterns of cooccurrence among the associative agnosias: implications for visual object representation. Cogn. Neuropsychol. 8, $1-19$.

Harris, A., and Nakayama, K. (2007). Rapid face-selective adaptation of an early extrastriate component in MEG. Cereb. Cortex 17, 63-70.

Hauk, O., Patterson, K., Woollams, A. Watling, L., Pulvermuller, F., and Rogers, T. T. (2006). When would you prefer a SOSSAGE to a SAUSAGE? [A] At about $100 \mathrm{msec}$. ERP correlates of orthographic typicality and lexicality in written word recognition. J. Cogn. Neurosci. 18, 818-832.

Hauk, O., and Pulvermuller, F. (2004). Effects of word length and frequency on the human event-related potential. Clin. Neurophysiol. 115, 1090-1103.

Holmes, A. P., Blair, R. C., Watson, J. D., and Ford, I. (1996). Nonparametric analysis of statistic images from functional mapping experiments. J. Cereb. Blood Flow Metab. 16, 7-22.

Itier, R. J., and Taylor, M. J. (2002). Inversion and contrast polarity reversal affect both encoding and recognition processes of unfamiliar faces: a repetition study using ERPs. Neuroimage 15, 353-372.

Itier, R. J., and Taylor, M. J. (2004). N170 or N1? Spatiotemporal differences between object and face processing using ERPs. Cereb. Cortex 14, 132-142.

Jacques, C., d'Arripe, O., and Rossion, B. (2007). The time course of the inversion effect during individual face discrimination. J. Vis. 7, 3.

Jacques, C., and Rossion, B. (2004). Concurrent processing reveals competition between visual representations of faces. Neuroreport 15, 2417-2421.

Jeffreys, D. A. (1989). A face-responsive potential recorded from the human scalp. Exp. Brain Res. 78, 193-202.

Jemel, B., Calabria, M., Delvenne, J. F., Crommelinck, M., and Bruyer, R. (2003). Differential involvement of episodic and face representations in ERP repetition effects. Neuroreport $14,525-530$.

Kovacs, G., Zimmer, M., Banko, E., Harza, I.,Antal,A., and Vidnyanszky, Z (2006). Electrophysiological correlates of visual adaptation to faces and body parts in humans. Cereb. Cortex 16, 742-753.

Lehmann, D., and Skrandies, W. (1980). Reference-free identification of components of checkerboard-evoked multichannel potential fields. Electroencephalogr. Clin. Neurophysiol. 48, 609-621.

Lund, K., and Burgess, C. (1996) Producing high-dimensional semantic spaces from lexical co-occurrence. Behav. Res. Methods Instrum. Comput. 28, 203-208.

Maurer,U.,Brandeis, D., and McCandliss, B. (2005a). Fast, visual specialization for reading in English revealed by the topography of the N170 ERP response. Behav. Brain Funct. 1, 13.

Maurer, U., Brem, S., Bucher, K., and Brandeis, D. (2005b). Emerging neurophysiological specialization for letter strings. J. Cogn. Neurosci. 17, 1532-1552.

Maurer, U., Brem, S., Kranz, F., Bucher, K. Benz, R., Halder, P., Steinhausen, H.-C. and Brandeis, D. (2006). Coarse neural tuning for print peaks when children learn to read. Neuroimage 33, 749-758.

Maurer, U., Zevin, J. D., and McCandliss, B. D. (2008). Leftlateralized N170 effects of visual expertise in reading: evidence from Japanese syllabic and logographic scripts. J. Cogn. Neurosci. 20, 1878-1891.

McCandliss, B. D., Cohen, L., and Dehaene, S. (2003). The visual word form area: expertise for reading in the fusiform gyrus. Trends Cogn. Sci. 7, 293-299.

Rossion, B., Collins, D., Goffaux, V., and Curran, T. (2007). Long-term expertise with artificial objects increases visual competition with early face categorization processes. J. Cogn. Neurosci. 19, 543-555.

Rossion, B., Gauthier, I., Tarr, M. J., Despland, P., Bruyer, R., Linotte, S., and Crommelinck, M. (2000). The N170 occipito-temporal component is delayed and enhanced to inverted faces but not to inverted objects: an electrophysiological account of facespecific processes in the human brain. Neuroreport 11, 69-74.

Rossion, B., and Jacques, C. (2008). Does physical interstimulus variance account for early electrophysiological face sensitive responses in the human brain? Ten lessons on the N170. Neuroimage 39, 1959-1979.

Rossion, B., Joyce, C. A., Cottrell, G. W., and Tarr, M. J. (2003). Early lateralization and orientation tuning for face, word, and object processing in the visual cortex. Neuroimage 20, 1609-1624.

Schweinberger, S. R., Pickering, E. C., Burton, A. M., and Kaufmann, J. M. (2002a). Human brain potential correlates of repetition priming in face and name recognition. Neuropsychologia 40, 2057-2073.

Schweinberger, S. R., Pickering, E. C., Jentzsch, I., Burton, A. M., and Kaufmann, J. M. (2002b). Eventrelated brain potential evidence for a response of inferior temporal cortex to familiar face repetitions. Brain Res. Cogn. Brain Res. 14, 398-409. 
Sergent, J. (1985). Influence of task and input factors on hemispheric involvement in face processing. J. Exp. Psychol. Hum. Percept. Perform. 11, 846-861.

Simon, G., Bernard, C., Largy, P., Lalonde, R., and Rebai, M. (2004). Chronometry of visual word recognition during passive and lexical decision tasks: an ERP investigation. Int. J. Neurosci. 114, 1401-1432.

Simon, G., Petit, L., Bernard, C., and Rebai, M. (2007). N170 ERPs could represent a logographic processing strategy in visual word recognition. Behav. Brain Funct. 3, 21.
Strik, W. K., Fallgatter, A. J., Brandeis, D., and Pascual-Marqui, R. D. (1998). Three-dimensional tomography of event-related potentials during response inhibition: evidence for phasic frontal lobe activation. Electroencephalogr. Clin. Neurophysiol. 108, 406-413.

Tanaka, J. W., and Curran, T. (2001). A neural basis for expert object recognition. Psychol. Sci. 12, 43-47.

Wong, A. C., Gauthier, I., Woroch, B., DeBuse, C., and Curran, T. (2005). An early electrophysiological response associated with expertise in letter perception. Cogn. Affect. Behav. Neurosci. 5, 306-318.

Conflict of Interest Statement: The authors declare that the research was conducted in the absence of any commercial or financial relationships that could be construed as a potential conflict of interest.

Received: 10 October 2008; paper pending published: 28 October 2008; accepted 12 November 2008; published online: 29 December 2008
Citation: Maurer $U$, Rossion B and McCandliss BD (2008) Category specificity in early perception: face and word $N 170$ responses differ in both lateralization and habituation properties. Front. Hum. Neurosci. (2008) 2:18. doi: 10.3389/neuro.09.018.2008

Copyright (c) 2008 Maurer, Rossion and McCandliss. This is an open-access article subject to an exclusive license agreement between the authors and the Frontiers Research Foundation, which permits unrestricted use, distribution, and reproduction in any medium, provided the original authors and source are credited. 